\title{
Effects of broccoli sprouts intake on oxidative stress, inflammation, microalbuminuria and platelet function in human volunteers: a cross-over study
}

\author{
E. Munters ${ }^{1}$, N. Pieters ${ }^{1}$, A. Cuypers ${ }^{1}$, J. Penders ${ }^{2,3}$, J. Vangronsveld $^{1}$ and T. Nawrot ${ }^{1}$ \\ ${ }^{1}$ Centre for Environmental Sciences, Hasselt University, Diepenbeek, Belgium, ${ }^{2}$ Hospital ZOL, Genk, Belgium and \\ ${ }^{3}$ Biomedical Research Institute, Hasselt University, Diepenbeek, Belgium
}

Broccoli sprouts contain a high content of glucosinolates ${ }^{(1)}$. These substances induce phase 2 enzymes, known for their protection against carcinogenesis $^{(2)}$, mutagenesis and other forms of toxicity of electrophiles and reactive forms of oxygen. We investigated the effects of the intake of broccoli sprouts on inflammation, microalbuminuria, platelet function and oxidative DNA damage.

The study included 23 patients aged between 22 and 35 years who had no history of chronic illnesses or other harmful diseases. They were randomised in a cross-over study with two treatments: $20 \mathrm{~g}$ broccoli sprouts or no broccoli sprouts for $4 \mathrm{~d}$. The two groups were crossed after 2 weeks. Platelet function was measured ex vivo with the PFA-100 platelet function analyser, which simulates a damaged blood vessel; we analysed the function of platelets in primary haemostasis under high shear conditions. Total and differential blood leucocytes were counted and oxidative DNA damage measured (8-hydroxy-2-deoxyguanosine [8-OH-2dG]) in urine from healthy volunteers.

Baseline values were: lymphocytes $\left(1000 \mu 1^{1}\right) 2.13 \pm 0.44$; monocytes $(\%) 5.90 \pm 1.07 ; 8-\mathrm{OH}-2 \mathrm{dG} / \mathrm{creatinine}(\mu \mathrm{g} / \mathrm{g}) 1.09 \pm 0.28$ and microalbumine/creatinine $(\mu \mathrm{g} / \mathrm{g}) 0.55 \pm 0.29$. These variations induced by broccoli sprout intake showed a $8.9 \%$ reduction $(P=0.029)$ in the amount of lymphocytes, a $11.9 \%$ reduction $(P=0.0093)$ in the percentage of monocytes, a $34.5 \%$ reduction $(P=0.016)$ in microalbumine/creatinine concentration and a $16.7 \%$ increase $(P=0.012)$ in $8-\mathrm{OH}-2 \mathrm{dG} /$ creatinine concentration. The intake of broccoli sprouts did not significantly influence platelet function action. Non-significant results were found in the assessment of blood and urine content in the control group.

Broccoli sprouts intake showed a significant reduction of lymphocytes, monocytes and microalbuminuria and a significant increase of 8-OH-2dG. Whether the increase in urinary $8-\mathrm{OH}-2 \mathrm{dG}$ reflects enhanced DNA repair or more repair because of increased oxidative DNA damage by broccoli sprouts must be further elucidated.

1. Shapiro TA, Fahey JW, Wade KL et al. (2001) Chemoprotective glucosinolates and isothiocyanates of broccoli sprouts: metabolism and excretion in humans. Cancer Epidemiol Biomarkers Prev 10(5), 501-508.

2. Verhagen H, de Vries A, Nijhoff WA et al. (1997) Effect of Brussels sprouts on oxidative DNA-damage in man. Cancer Lett 114(1-2), 127-130. 